University of Nebraska - Lincoln

DigitalCommons@University of Nebraska - Lincoln

Timothy J. Gay Publications

Research Papers in Physics and Astronomy

December 1996

\title{
An inline optical electron polarimeter
}

K. W. Trantham

University of Nebraska - Lincoln

Timothy J. Gay

University of Nebraska - Lincoln, tgay1@unl.edu

R. J. Vandiver

University of Missouri-Rolla, Rolla, Missouri

Follow this and additional works at: https://digitalcommons.unl.edu/physicsgay

Part of the Physics Commons

Trantham, K. W.; Gay, Timothy J. ; and Vandiver, R. J., "An inline optical electron polarimeter" (1996).

Timothy J. Gay Publications. 42.

https://digitalcommons.unl.edu/physicsgay/42

This Article is brought to you for free and open access by the Research Papers in Physics and Astronomy at DigitalCommons@University of Nebraska - Lincoln. It has been accepted for inclusion in Timothy J. Gay Publications by an authorized administrator of DigitalCommons@University of Nebraska - Lincoln. 


\section{An inline optical electron polarimeter}

K. W. Trantham ${ }^{\text {a) }}$ and T. J. Gay ${ }^{\text {b) }}$

Behlen Laboratory of Physics, University of Nebraska, Lincoln, Nebraska 68588-0111

R. J. Vandiver ${ }^{\mathrm{c})}$

Physics Department, University of Missouri-Rolla, Rolla, Missouri 65401

(Received 8 May 1996; accepted for publication 9 September 1996)

The design and operation of a simple inline optical electron polarimeter is presented. It is based on exchange excitation of ground state neon atoms. The electron polarization is determined from the degree of circular polarization of the subsequent $2 p^{5} 3 p^{3} D_{3} \rightarrow 2 p^{5} 3 s^{3} P_{2}$ (6402 $\AA$ ) fluorescence. This device can characterize both longitudinally and transversely polarized electron beams in a nondestructive fashion, and is inexpensive and easily constructed. (C) 1996 American Institute of Physics. [S0034-6748(96)02112-0]

\section{INTRODUCTION}

In electron-atom collision experiments, it is desirable to start with electrons in a well-defined spin state just as it is desirable to have a well-defined beam energy. It is therefore necessary to measure the electron polarization. Other experiments may require measuring the polarization of scattered electrons. Many different types of electron polarimeters have been developed. ${ }^{1}$ The most common of these is the Mott polarimeter. We have constructed another type of polarimeter based on the excitation of an atomic target. The polarization of the optical radiative decay determines the degree of spin polarization of the electron beam. This "optical" polarimeter has some advantages compared to the Mott polarimeter.

An electron beam is said to be polarized if there exists any axis of quantization $\hat{\imath}$ for which the two possible spin states are not equally populated. The magnitude of polarization along that axis is

$$
P(\hat{\imath})=\frac{N_{\uparrow}-N_{\downarrow}}{N_{\uparrow}+N_{\downarrow}},
$$

where $N_{\uparrow}\left(N_{\downarrow}\right)$ refers to the number of electrons whose spins are parallel (antiparallel) to the chosen direction. If the direction of maximum polarization $\mathbf{P}$ is parallel or antiparallel to the electron momentum $\mathbf{k}$, the polarization is longitudinal; if this direction is perpendicular to $\mathbf{k}$ the electrons are transversely polarized.

The Mott polarimeter is based on electron scattering asymmetries caused by spin-orbit interactions with a heavy nucleus. ${ }^{2}$ By measuring the number of electrons elastically scattering at angles $\pm \theta$ in a plane perpendicular to the initial electron polarization, one defines the scattering asymmetry

$$
A \equiv \frac{N_{+}-N_{-}}{N_{+}+N_{-}} .
$$

Then $P$ can be found from

$$
A=S(\theta) P,
$$

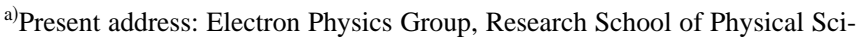
ences, The Australian National University, Canberra ACT 0200, Australia.

${ }^{b)}$ Electronic mail: tgay@unl.edu

${ }^{c}$ Present address: P.O. Box 182, Malden, MO 63863.
}

where $S(\theta)$ is the Sherman function, ${ }^{2}$ or analyzing power of the device, and is calculable for elastic scattering from a single nucleus. Since single elastic events rarely occur in a typical scattering target, the "effective Sherman function," $S_{\text {eff }}$, which takes into account the effects of scattering from a thick target and other instrumental effects, must be measured in a calibration experiment. In addition to the calibration requirement, another disadvantage of standard Mott polarimetry is that it can only measure transversely polarized beams. The use of a spin rotator is therefore required as most spin polarized electron sources produce longitudinally polarized beams. Other disadvantages can include large apparatus size and high operating voltages $(20-100 \mathrm{kV})$.

An alternative polarimeter is based on the impact excitation of an atomic target and measuring the degree of circular polarization in the subsequent optical decay. ${ }^{3-5}$ The incident electron polarization information is conveyed to the atom by exchange excitation from a singlet ground state to an excited triplet state. Since it is the orientation of the angular momentum of the atom that gives rise to circularly polarized fluorescence, one has to make a judicious choice of which angular momentum states to monitor in the optical decay process. In the first demonstration of optical electron polarimetry, the $4 s^{2}{ }^{1} S_{0}$ ground state of $\mathrm{Zn}$ was excited to the $4 s 5 s^{3} S_{1}$ level. ${ }^{6,7}$ The fluorescence from the decay to the $4 s 5{ }^{3} P_{J}$ states was monitored. This method requires that the ${ }^{3} S_{1} \rightarrow{ }^{3} P_{J}$ multiplet be resolved to observe circular polarization. A polarimetric expression can be obtained of the form

$$
S / I=\Lambda P,
$$

where $S / I$ is the relative Stokes parameter for the degree of circular polarization. The analyzing power, $\Lambda$, of this optical polarimeter is directly calculable and is generally higher $(\sim 0.7)$ than that of Mott polarimeters $(\sim 0.4)$. The main disadvantages of using a $\mathrm{Zn}$ target are the necessity of resolving the fine structure of the decay fluorescence and the experimental difficulty of working with heavy metal vapors. Similar difficulties with $\mathrm{Hg}$ are compounded by the depolarizing effects of negative ion resonances. ${ }^{8}$ These problems are solved by using helium as the target gas. ${ }^{9-11}$ With the $\mathrm{He}$ polarimeter, one monitors the decay $3{ }^{3} P_{J} \rightarrow 2{ }^{3} S$. Here the $J$ multiplet need not be resolved because the upper level has a 


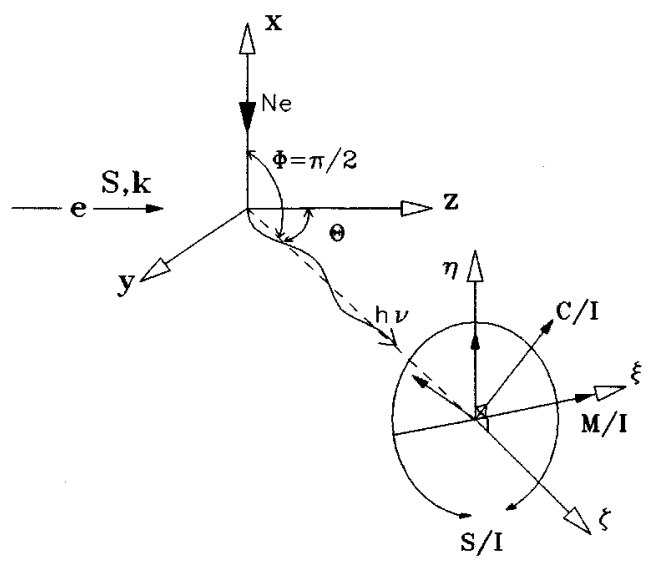

FIG. 1. Polarimeter collision geometry.

nonzero orbital angular momentum. Furst et al. $^{3}$ have shown that an optical electron polarimeter can also be based on any of the heavy noble gases.

We have constructed a simple electron polarimeter that uses a neon target, which by virtue of its geometry can measure either longitudinal or transverse polarization. This device was designed to be used as an inline polarimeter to perform systematic checks on the polarization of our electron source being used for another experiment. Polarized electrons excite the neon $2 p^{5} 3 p^{3} D_{3}$ level from the $2 p^{6}{ }^{1} S_{0}$ ground state. The polarization of the photons $(\lambda=6402 \AA)$ from the decay to the $2 p^{5} 3 s^{3} P_{2}$ state is used to determine the spin polarization of the incident electrons. The excitation threshold for the ${ }^{3} D_{3}$ state is $18.5 \mathrm{eV}$. At $19.6 \mathrm{eV}$, it begins to be possible to excite other states which can cascade through the ${ }^{3} D_{3}$ level. This is not desirable as these higher states will not generally contain well-characterized information regarding the spin of the impact electron. ${ }^{3,4}$ However, because of the energy dependance of the various excitation cross sections, the effect of cascades should not significantly effect the polarization measurements until the incident beam energy is greater than $22 \mathrm{eV}$.

In the present discussion, we consider longitudinal polarization with the incident electron momentum defining the $z$ axis as shown in Fig. 1. An effusive neon beam directed along $-\hat{x}$ is crossed with the electron beam. Fluorescence is observed along the $\zeta$ axis at polar angle $\theta=135^{\circ}$ and azimuthal angle $\phi=90^{\circ}$. The $(\xi, \eta, \zeta)$ coordinate system shown in Fig. 1 is used to define the polarization of the light with relative Stokes parameters given as

$$
M / I=\frac{I(0)-I(\pi / 2)}{I(0)+I(\pi / 2)}, \quad C / I=\frac{I(\pi / 4)-I(3 \pi / 4)}{I(\pi / 4)+I(3 \pi / 4)},
$$

and

$$
S / I=\frac{I\left(\sigma^{+}\right)-I\left(\sigma^{-}\right)}{I\left(\sigma^{+}\right)+I\left(\sigma^{-}\right)},
$$

where $I(\delta)$ is the intensity of light polarized along an axis making an angle $\delta$ with the $\xi$ axis. Right and left handed circularly polarized light is denoted by $\sigma^{ \pm}$. It can thus be shown $^{3,5}$ that for longitudinal electron polarization in the geometry of Fig. 1 that

$$
S / I=(\sqrt{2} / 3)\left[1-\frac{17}{9} M / I\right] P .
$$

If the electron polarization is transverse along $\hat{y}$ (but not $\hat{x}$ ) in Fig. 1, a different expression would be obtained for $P$, but no experimental geometry changes would be required (see e.g., Ref. 3 and references therein). In obtaining Eq. (6), it is assumed that the ${ }^{3} D_{3}$ state is well $L-S$ coupled and that effects such as Mott scattering can be ignored. ${ }^{12}$ In this case, the analyzing power expression in Eq. (6) is exact and purely the result of Clebsh-Gordon algebra, unlike the Sherman function which requires dynamical calculation. Also, $C / I$ will be identically zero. (This latter null condition can be used, conversely, to check the validity of the assumptions made above.) The threshold value of $M / I$ for the geometry of Fig. 1 is kinematically required to be $0.28 .^{13}$ Thus the analyzing power of this device is $\approx 0.22$. This is lower than that for typical high energy $(>100 \mathrm{keV})$ Mott polarimeters because of our observation angle. If the fluorescence polarization was measured parallel to $\mathbf{P}(\theta=0$ for longitudinal polarization), $\Lambda$ would be higher $(\sim 0.7)$.

\section{APPARATUS}

Our polarimeter is quite simple mechanically and is shown in Fig. 2. Its vacuum requirements are not stringent. The vacuum chamber is a standard glass cross commercially available for food processing and sewer use. The cross has been modified by adding a $21 / 2$ in. Pyrex window at an angle of $45^{\circ}$ thus providing an approximately flat viewing port. The chamber is connected to a diffusion pump and other chambers with elastomer seals. A typical base pressure is $\sim 5 \times 10^{-7}$ Torr.

The electrons are transported to and focused on the neon target chamber using a five-element afocal cylindrical lens system as described by Heddle. ${ }^{14}$ An identical lens train transports the beam from the gas target to the next chamber downstream. Each lens is a simple cylinder machined from aluminum and then coated with a thin, uniform layer of aqueous colloidal graphite. The lens elements are mounted to a track from which they are electrically separated by glass tubes. The tubes also serve to axially align the lens elements. Even at the relatively low electron energies we used, deleterious beam handling effects due to patch fields, charging insulators, etc. were not observed. The target chamber was constructed in the same manner as the lens elements. Target gas is introduced via a suitable tube with a large length-todiameter ratio. Although a capillary array could be used, we found a brass tube (diameter $0.7 \mathrm{~mm}$ and length $\sim 12 \mathrm{~cm}$ ) to work sufficiently well. The target has large holes fitted with a brass screen to allow swift pumping while maintaining a constant electric field in the interaction region. A smaller additional hole is provided to monitor the fluorescence, as illustrated in Fig. 2.

Special attention must be paid to the reduction of magnetic fields along the electron beam line and in the target region. Magnetic fields can cause the electron's spin to precess prior to the collision region. Also, independent of the electron's spin, the atomic alignment and orientation will precess in a magnetic field before the atomic state decays 

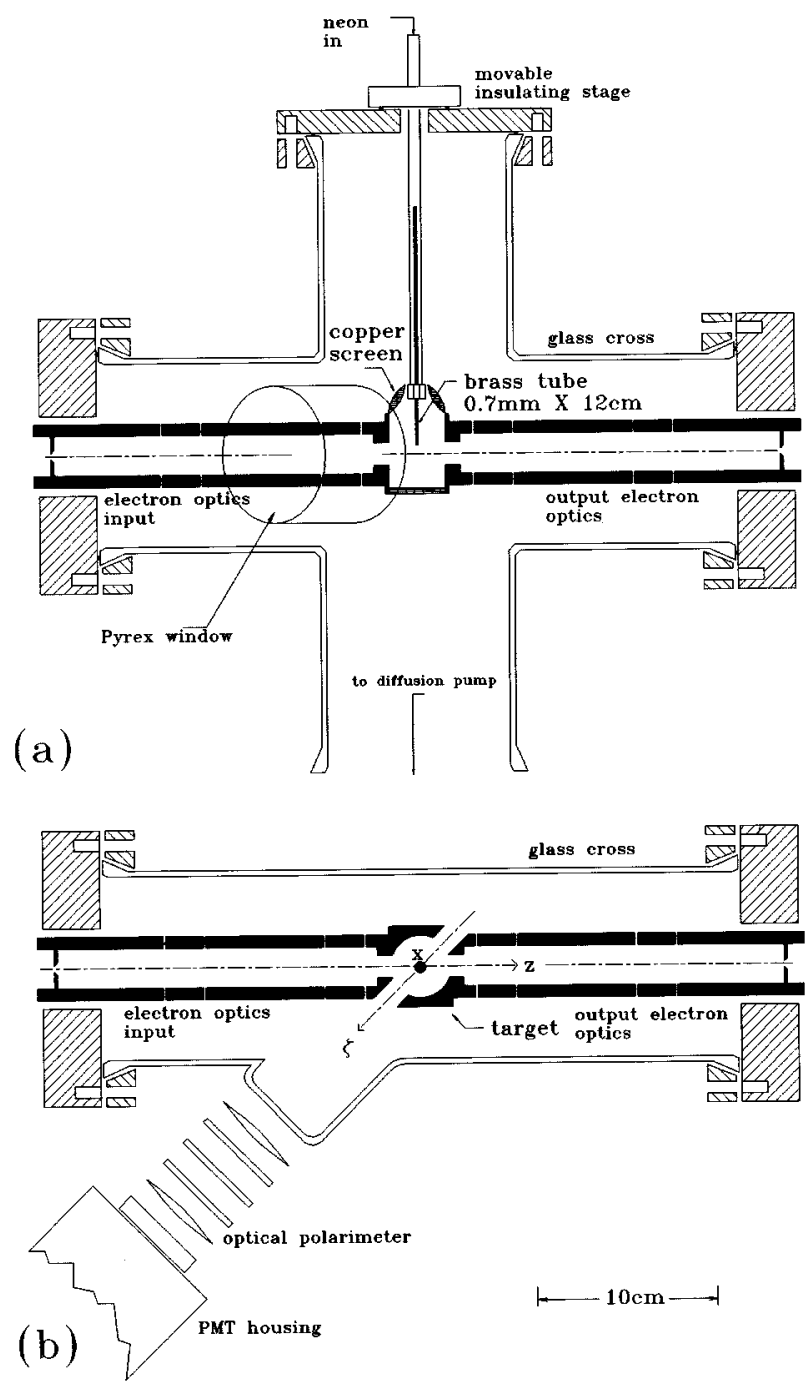

FIG. 2. Noble gas electron polarimeter showing apparatus side (a) and top (b) views.

(Hanle effect). Both of these mechanisms change the effective fluorescence detection angle. Thus the measured Stokes parameters will not correspond to the assumed collision geometry, and an erroneous value of $P$ will be obtained. To control these unfavorable effects, we have oriented our beam line along magnetic north. A large pair of rectangular coils are used to minimize the vertical field along the beam. The residual longitudinal field has no effect for our geometry. Also, the construction of the neon target, gas injection hardware, etc., was made with nonmagnetic materials. Even "nonmagnetic" stainless steel was deemed unacceptable as machining processes can slightly magnetize this material.

At the heart of our apparatus is the optical polarimeter. In principle, the linear polarization in Eq. (6), $M / I$, only needs to be measured once. Then one could make routine measurements of $P$ by simply measuring $S / I$. However, as a diagnostic, we were interested in simultaneous measurement of all three relative Stokes parameters. The linear polarization fraction $M / I$ has a kinematically required threshold value so its measurement near $18.6 \mathrm{eV}$ serves as a check of the apparatus. The measurement of $C / I$ also serves this func-

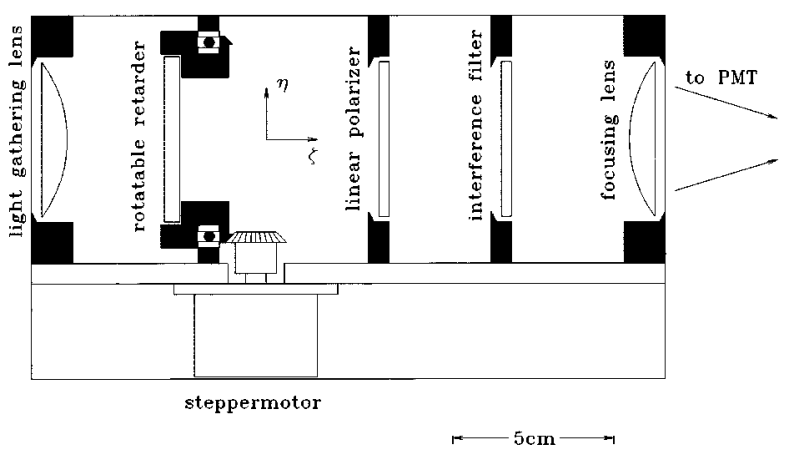

FIG. 3. Cross-sectional view of the photon polarimeter.

tion, since it should be identically zero in all cases barring a breakdown of the basic assumptions required to derive Eq. (6). To this end, we have patterned our optical polarimeter after that described by Berry et al., ${ }^{15}$ and measure all three relative Stokes parameters simultaneously.

As illustrated in Fig. 3, the optical train consists of a light-gathering lens, an achromatic quarter-wave retarder (manufactured by Meadowlark Optics), a linear polarizer (Rolyn No. 65.5305), an interference filter for the neon transition of interest, and finally a lens to focus the fluorescence onto the GaAs photocathode of a Hamamatsu R943-02 photomultiplier tube (PMT). Our interference filter (Andover Corporation) has a center-pass wavelength of $6403 \AA$ and transmission full width at half-maximum of $9 \AA$. The linear polarizer transmission axis is fixed and makes some known angle $\alpha$ with respect to the $\xi$ axis (Fig. 1). The retarder is rotatable, with the fast axis making angle $\beta$ relative to the $\xi$ axis. In this configuration, the transmitted light to the PMT is given by

$$
\begin{aligned}
I_{t}= & \frac{1}{2}\left[I+\frac{1}{2} M \cos (2 \alpha)+\frac{1}{2} C \sin (2 \alpha)\right] \\
& +\frac{1}{2} S \sin \left(2 \alpha-2 \beta-2 \beta_{0}\right)+\frac{1}{4} M \cos \left(2 \alpha-4 \beta-4 \beta_{0}\right) \\
& -\frac{1}{4} C \sin \left(2 \alpha-4 \beta-4 \beta_{0}\right),
\end{aligned}
$$

where $\beta_{0}$ is the offset of the real fast axis from its assumed position. Measurement of the photon polarization is done by rotating the retarder with a stepper motor and recording the PMT counts at each $\beta$. Both of these tasks are handled with a computer. We typically rotate the retarder in $4.5^{\circ}$ steps for three revolutions. By fitting the data to a function of the form

$$
\begin{aligned}
I_{t}= & X_{0}+X_{1} \sin \left(2 \alpha-2 \beta-2 \beta_{0}\right)+X_{2} \cos \left(2 \alpha-4 \beta-4 \beta_{0}\right) \\
& +X_{3} \sin \left(2 \alpha-4 \beta-4 \beta_{0}\right),
\end{aligned}
$$

the parameters $I, M, C$, and $S$ (and thus $M / I, C / I$, and $S / I$ ) are found from the fitting coefficients $X_{i}$. The errors of the Stokes parameters are derived from the standard errors of these coefficients.

Due to possible errors in marking the axes of the polarizing elements or in inserting them in their respective holders, $\alpha$ and $\beta_{0}$ are measured in situ. This is accomplished by rewriting the $S$ term in Eq. (7) as

$$
\frac{1}{2} S\left[\sin \left(2 \alpha-2 \beta_{0}\right) \cos (2 \beta)-\cos \left(2 \alpha-2 \beta_{0}\right) \sin (2 \beta)\right] .
$$




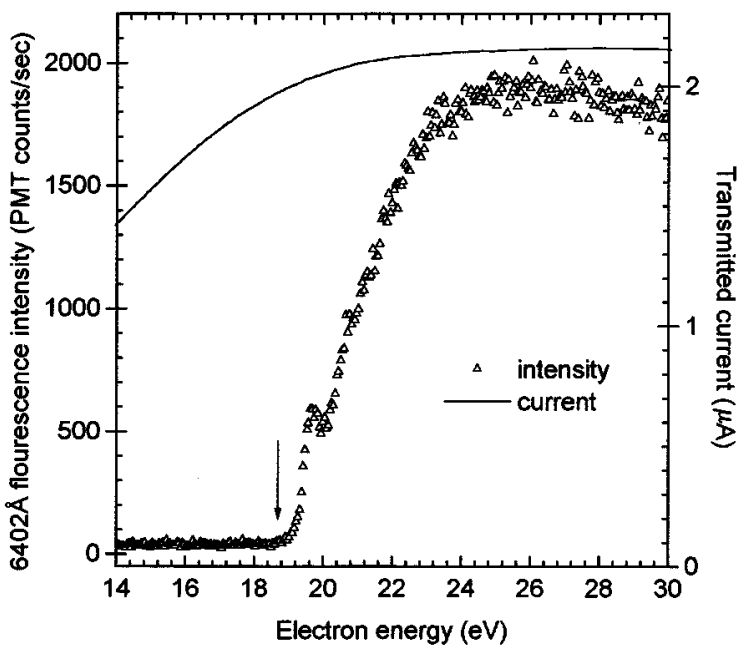

FIG. 4. Excitation function used to determine energy scale relative to target potential (see the text). Arrow indicates onset of optical signal above background.

Then, two sets of data are taken with the optical polarimeter observing strongly circularly polarized light. Either helicity of light will work and less than perfect polarization only effects the error estimate. Any moderate degree of linear polarization in the test light will not affect the measurement, except in error analysis, because it is associated with the wrong "frequency", (in $\beta$ ) in Eq. (7). The first set of data is taken with the linear polarizer in its usual position (the " + ," run). Then the polarizer is flipped about the $\eta$ axis, so $\alpha \rightarrow$ $(-\alpha)$, and a second set of data is taken (the " - ," run). Our polarizer holder is designed to be mounted in the optical polarimeter in either direction while holding the polarizing element fixed relative to itself, thus ensuring repeatability of $\alpha$. Each set of data is fit with the function

$$
\begin{aligned}
I_{t}= & A_{0}+A_{1}^{ \pm} \sin (2 \beta)+A_{2} \cos (4 \beta)+A_{3} \sin (4 \beta) \\
& +A_{4}^{ \pm} \cos (2 \beta),
\end{aligned}
$$

where the \pm superscript distinguishes the run. Hence, the angles $\alpha$ and $\beta_{0}$ are found from

$\frac{1}{2} \arctan \left(\frac{A_{1}^{+}}{A_{4}^{+}}\right)=\alpha-\beta_{0} \quad$ and $\quad \frac{1}{2} \arctan \left(\frac{A_{1}^{-}}{A_{4}^{-}}\right)=-\alpha-\beta_{0}$.

Note that since this method relies on changes of phase in the $\sin (2 \beta)$ term of Eq. (7) when $\alpha$ is reversed, changes in background or light source intensity between runs will not affect the results.

\section{SAMPLE DATA}

To test and characterize the apparatus, several other measurements must be made. The most fundamental one involves establishing an energy scale. Due to contact potential differences, the energy of the electrons is not simply the electric charge times the potential difference between the target and electron emitter. By changing the target potential and recording the PMT count rate, one obtains an "excitation function," shown in Fig. 4. Changing the target potential

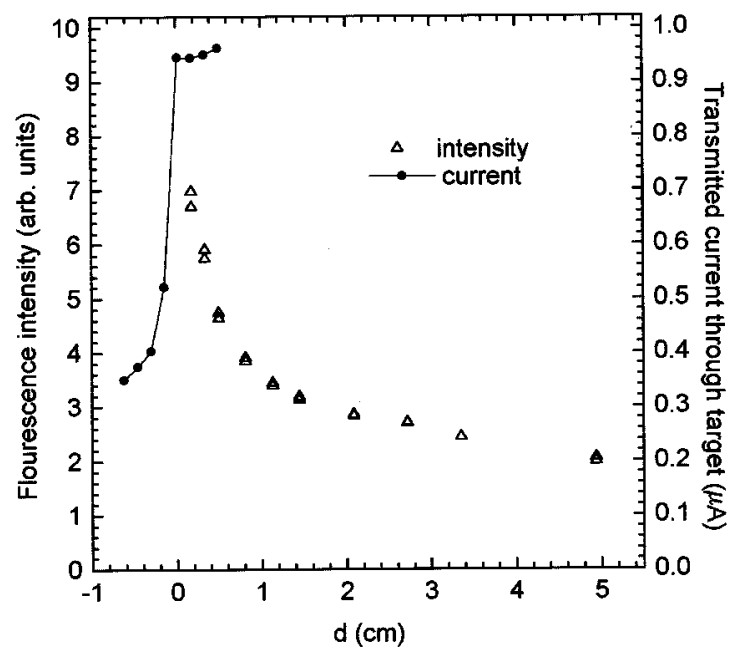

FIG. 5. Fluorescence intensity as a function of the distance of the gas needle tip to the electron beam, $d$. Transmitted current through the target is used to determine $d=0$ position.

also changes the transmitted current, as shown in Fig. 4, and, to a lesser extent, the electron beam-neon jet overlap. Thus the excitation function we measure is not the true optical excitation cross section for the ${ }^{3} D_{3}$ state, but will adequately serve the purpose of setting an energy scale. The excitation threshold for this state is $18.55 \mathrm{eV}$, so identifying the onset of $6402 \AA$ fluorescence will establish an energy scale relative to the target potential. This is identified by the first increase in PMT count rate above background, as indicated by the arrow. Thus this procedure associates the electron beam's upper tail of its energy distribution with the threshold value of $18.55 \mathrm{eV}$. The electron source used for this study is based on photoemission from GaAs and typically has an energy width of $<200 \mathrm{meV}$.

Figure 4 can also be used to estimate the polarimeter's overall efficiency, i.e., the detected count rate divided by the incident particle current. At the peak of the excitation function, we obtain a PMT count rate of about $1 \mathrm{kHz}$ for every microampere of current incident on the target. This corresponds to an efficiency of the order $10^{-10}$, as compared with efficiencies ranging between $10^{-1}$ and $10^{-7}$ for polarimeters based on Mott scattering. ${ }^{2}$ Thus optical polarimeters of this type are really only useful for the analysis of primary electron beams.

It is important to understand how the gas target needle position affects the fluorescence intensity and polarization. Figure 5 shows the intensity of the fluorescence (measured at the excitation function peak) as the gas needle is raised from the electron beam. The drop in intensity results from a drop in local neon number density at the electron collision region. Also shown is the transmitted electron current through the target, substantiating the origin of the abscissa. The relative Stokes parameters were also measured at different heights, but no position dependence was observed.

Further checks of the apparatus included measurements of $S / I$ and $C / I$ when the electron polarization was flipped. We found that $S / I$ reversed sign exactly, within statistical uncertainties, indicating that no significant circular instru- 

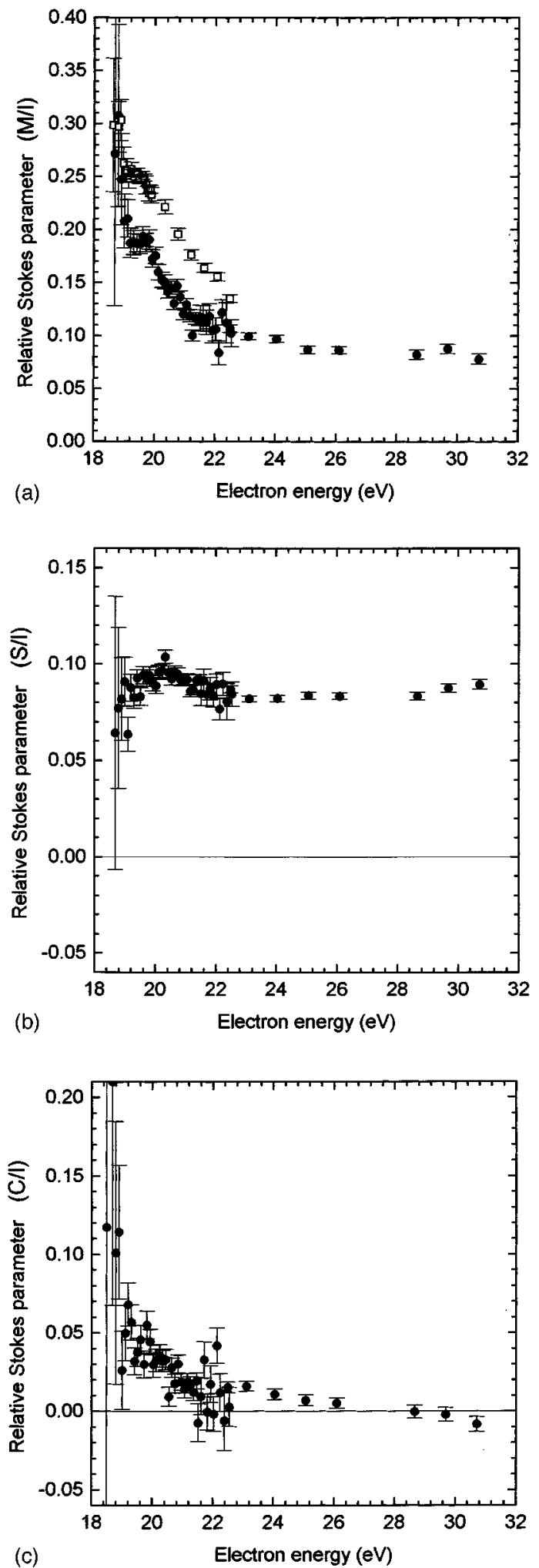

FIG. 6. (a) Relative Stokes parameter $M / I$ measured using (i) rotation of the quarter-wave plate only (open squares), and (ii) rotation of the quarter-wave plate and linear polarizer together (solid squares). See the text. (b) Relative Stokes parameter $S / I$. (c) Relative Stokes parameter $C / I$.

mental polarization was present. The linear polarization $C / I$, however, was generally nonzero, with a magnitude of several percent. These values did not change when electron polarization was reversed, showing that LS coupling [and hence Eq.

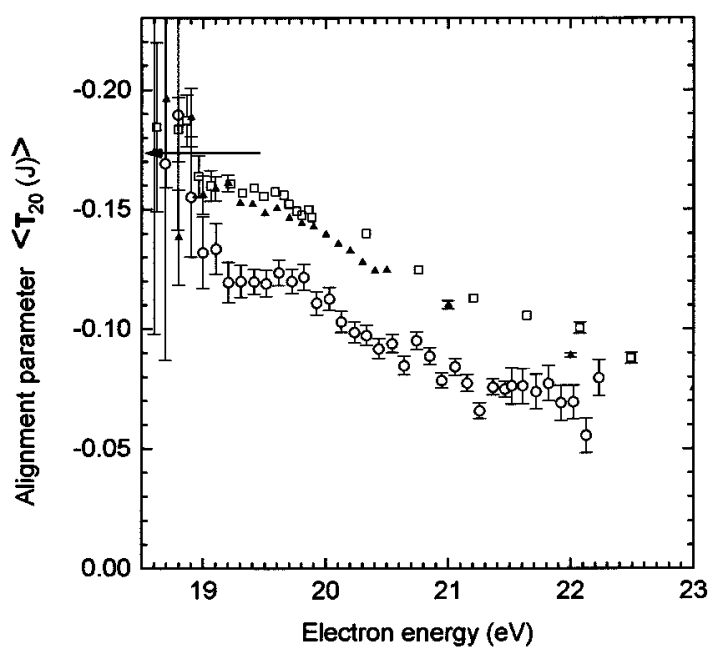

FIG. 7. Comparison of the alignment parameter $\left\langle T_{20}(J)\right\rangle$ computed from present $M / I$ data (open squares, rotating quarter-wave plate, and polarizer; open circles, rotating quarter-wave plate only) and that of Ref. 3 (solid triangles). The arrow indicates the kinematically required threshold value.

(6) ] held for the system, ${ }^{12}$ but also indicating the presence of a residual linear polarization instrumental asymmetry. We believe this to be a manifestation of the "approximately" flat window mentioned in the apparatus description. We typically took data when the bottom of the target needle was $\sim 3$ $\mathrm{mm}$ above the electron beam, with a neon partial pressure of $6 \times 10^{-6}$ Torr (as indicated by our chamber ionization gauge).

As mentioned above, polarization measurements requiring no calibration must be made at energies below that of the threshold for the first cascading level. However, for better counting statistics, $S / I$ can be measured at the peak of the excitation function. Electron polarization is then determined by normalizing to previous measurements made near threshold. Shown in Figs. 6(a)-6(c) are measurements of $M / I, S / I$, and $C / I$ as a function of incident electron energy. Background light in these measurements is accounted for by measuring the $I_{t}(\beta)[\mathrm{Eq} .(8)]$ at $50-250 \mathrm{meV}$ below the $6402 \AA$ threshold and subtracting these values from the $I_{t}(\beta)$ made above threshold before Eq. (8) is regression analyzed. This procedure accounts for a background that may be polarized.

There are two sets of $M / I$ data shown in Fig. 6(a). The first set was taken with the algorithm outlined above [using Eq. (8)]. The second set was taken by placing the linear polarizer in front of the quarter-wave plate so that the light transmitted to the PMT was circularly polarized, and toggling this pair together between $\alpha=0^{\circ}$ and $\alpha=90^{\circ}$. Then, $M / I$ is computed from

$$
M / I=\frac{I(0)-I(90)}{I(0)+I(90)} .
$$

We found with this apparatus that reproducability of $M / I$ measurements using these two methods was not better that about 0.05 (absolute). While both data sets in Fig. 6(a) have the correct threshold value (within our statistical uncertainties), they are systematically different from each other in the region above threshold. We attribute these problems, which did not seem to affect the other Stokes parameter mea- 


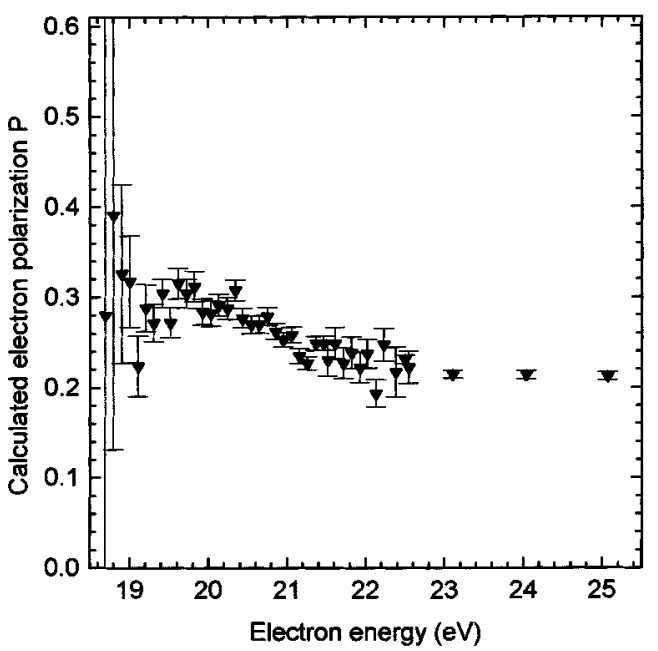

FIG. 8. Computed electron polarization $P$ using Eq. (6), as a function of incident electron energy $E$.

surements, to instrumental linear polarization of the $45^{\circ}$ window that may have been highly sensitive to the position of the fluorescence source, i.e., the incident beam trajectory. Another possible cause could be spurious reflections of primary fluorescence, which might affect linear polarizations more than circular ones. We can compare the current $M / I$ results in Fig. 6(a) with previous measurements (for which reproducibility was not a problem) made in our laboratory at a right angle to the incident beam. ${ }^{3}$ This is done by converting both data sets into their equivalent $\left\langle T(J)_{20}\right\rangle$ alignment multiple moments. ${ }^{16}$ The results are shown in Fig. 7, and the agreement between at least one of our current data sets and the results of Furst $e t$ al. $^{3}$ is acceptable. We note that a 0.05 uncertainty in $M / I$ results in about a 0.02 absolute uncertainty in $P$. As indicated by Fig. 6(c), $C / I$ is not generally zero. Again, we believe the poor optical properties of the vacuum window to be the problem.

Plotted in Fig. 8 is the value for $P$ obtained using Eq. (6) with the data of Figs. 6(a) and 6(b). Only in the region between 18.55 and $19.6 \mathrm{eV}$ are the results free from cascading. From data in this region, we obtain $29.1 \%$ polarization with a statistical uncertainty of $0.7 \%$ for our bulk GaAs electron source. This is consistent with other GaAs sources operated under similar conditions.
In conclusion, we have constructed a practical inline optical electron polarimeter that uses a neon target. While this particular apparatus has some shortcomings with regard to the quality of the optical train and could not be used as a high precision electron polarimeter, it is suitable to monitor the output beam of a polarized electron source. It is simple to use and has relatively modest apparatus requirements. We are currently building a new noble gas electron polarimeter with which we intend to make very precise measurements. Such a polarimeter is free of most difficulties encountered with polarimeters using targets of $\mathrm{Hg}, \mathrm{Cd}$, or $\mathrm{Zn}$, and does not require calibration.

\section{ACKNOWLEDGMENTS}

The authors gratefully acknowledge useful discussions with J. E. Furst, B. G. Birdsey, and the late L. D. Schearer. This work was supported by the National Science Foundation under Grant No. PHY-9504350.

${ }^{1}$ T. J. Gay in Atomic, Molecular, and Optical Physics: Charged Particles, edited by F. B. Dunning and R. G. Hulet (Academic, New York, 1995), Vol 29A.

${ }^{2}$ T. J. Gay and F. B. Dunning, Rev. Sci. Instrum. 36, 881 (1992).

${ }^{3}$ J. E. Furst, W. M. K. P. Wijayaratna, D. H. Madison, and T. J. Gay, Phys. Rev. A 47, 3775 (1993).

${ }^{4}$ T. J. Gay, J. E. Furst, K. W. Trantham, and W. M. K. P. Wijayaratna, Phys. Rev. A 53, 1623 (1996).

${ }^{5}$ K. W. Trantham, M.S. thesis, University of Missouri (Rolla), 1993 (unpublished).

${ }^{6}$ P. S. Farago and J. S. Wykes, J. Phys. B 2, 747 (1969); J. Wykes, ibid. 4, 191 (1971).

${ }^{7}$ M. Eminyan and G. Lampel, Phys. Rev. Lett. 45, 1171 (1980).

${ }^{8}$ A. Wolcke, K. Bartschat, K. Blum, H. Borgmann, G. F. Hanne, and J. Kessler, J. Phys. B 16, 639 (1983).

${ }^{9}$ T. J. Gay, J. Phys. B 16, L553 (1983).

${ }^{10}$ M. Uhrig, A. Beck, J. Goeke, F. Eschen, M. Sohn, G. F. Hanne, K. Jost, and J. Kessler, Rev. Sci. Instrum. 60, 872 (1989); J. Goeke, J. Kessler, and G. F. Hanne, Phys. Rev. Lett. 59, 1413 (1987).

${ }^{11}$ I. Humphry, J. F. Williams, and E. L. Heck, J. Phys. B 20, 367 (1987).

${ }^{12}$ J. E. Furst, T. J. Gay, and W. M. K. P. Wijayaratna, J. Phys. B 25, 1089 (1992).

${ }^{13}$ U. Fano and J. Macek, Rev. Mod. Phys. 45, 553 (1973).

${ }^{14}$ D. W. O. Heddle, Electrostatic Lens Systems (Hilger, Bristol, 1991).

${ }^{15}$ H. G. Berry, G. Gabrielse, and A. E. Livingston, Appl. Opt. 16, 3200 (1977).

${ }^{16}$ K. Blum, Density Matrix Theory (Plenum, New York, 1981). 